\title{
Atopic dermatitis is not associated with SARS-CoV-2 outcomes
}

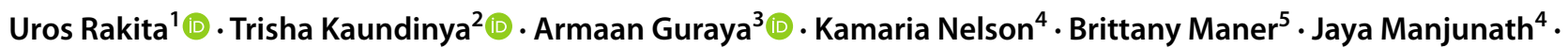 \\ Gabrielle Schwartzman ${ }^{4}$ Brittany Lane ${ }^{6} \cdot$ Jonathan I. Silverberg ${ }^{4,7}$ (I)
}

Received: 13 July 2021 / Accepted: 20 August 2021 / Published online: 17 September 2021

(c) The Author(s), under exclusive licence to Springer-Verlag GmbH Germany, part of Springer Nature 2021

\begin{abstract}
Atopic dermatitis is characterized by immune dysregulation, which may predispose toward worse COVID-19 outcomes. We conducted a retrospective cohort study to investigate the relationship of atopic dermatitis with COVID-19 symptom severity, hospitalization, length of hospital stay, requirement for oxygen therapy, long-term morbidity and mortality. Multivariable logistic regression models were constructed to examine the impact of atopic dermatitis (independent variable) on COVID-19 symptom severity, hospitalization, length of hospital stay, requirement for oxygen therapy, long-term morbidity and mortality (dependent variables). SARS-CoV-2 positive adult patients with diagnosed AD had similar odds of hospitalization (adjusted odds ratio [95\% confidence interval]: 0.51 [0.20-1.35]), acute level of care at initial medical care $(0.67$ [0.35-1.30]), severecritical SARS-CoV-2 (0.82 [0.29-2.30]), requirement of supplemental non-mechanical oxygen therapy (1.33 [0.50-3.58]), extended hospital stay (2.24 [0.36-13.85]), lingering COVID-19 symptoms (0.58 [0.06-5.31]) and COVID-19 death (0.002 $[<0.001->999])$ compared to patients without AD. Our findings suggest AD is not an independent risk factor for COVID19 severity or complications.
\end{abstract}

Keywords Atopic dermatitis · COVID-19 $\cdot$ SARS-CoV-2 $\cdot$ Epidemiology

\section{Introduction}

Atopic dermatitis (AD) is a chronic inflammatory skin disease associated with systemic T-helper 2 activation and respiratory comorbidities, e.g. asthma and rhinitis. Concern

Jonathan I. Silverberg

Jonathanisilverberg@gmail.com

1 Chicago Medical School, Rosalind Franklin University, North Chicago, IL 60064, USA

2 Northwestern Feinberg School of Medicine, Chicago, IL 60611, USA

3 Midwestern University Chicago College of Osteopathic Medicine, Chicago, IL 60515, USA

4 Department of Dermatology, George Washington School of Medicine, Washington, DC 20052, USA

5 Ross University School of Medicine, St. Michael, Barbados, BB 11093, USA

6 Michigan State University College of Human Medicine, East Lansing, MI 48824, USA

7 George Washington University School of Medicine and Health Sciences, Suite 2B-425, 2150 Pennsylvania Avenue NW, Washington, DC 20037, USA exists regarding potential for poorer COVID-19 outcomes in $\mathrm{AD}$ patients, though previous studies had mixed findings [1-5]. We investigated the relationship between AD and COVID-19 outcomes in adults.

\section{Methods}

The study was approved by the George Washington University (GWU) institutional review board. We retrospectively analyzed data from GWU medical records for patients treated for SARS-CoV-2. Sociodemographic traits were compared between those with vs. without AD and severecritical vs. mild-moderate COVID-19 using chi-squared and student's $t$ test for categorical and continuous variables, respectively (Table 1). Binary logistic regression models were constructed with COVID-19 outcomes as dependent variables (acuity level of initial medical contact, hospitalization, hospitalization duration, COVID-19 symptom severity, requirement of supplemental oxygen therapy, mortality and 
Table 1 Sociodemographic and other health-related associations related to diagnosis of atopic dermatitis

\begin{tabular}{|c|c|c|c|c|c|c|c|c|c|c|c|}
\hline \multirow[t]{3}{*}{ Variable } & \multicolumn{6}{|c|}{ Atopic dermatitis } & \multicolumn{5}{|c|}{ COVID-19 severity } \\
\hline & \multicolumn{2}{|c|}{ Yes } & \multicolumn{2}{|l|}{ No } & \multicolumn{2}{|c|}{$P$ value* } & \multicolumn{2}{|c|}{ Asymptomatic-mild } & \multicolumn{2}{|c|}{ Severe-critical } & \multirow[t]{2}{*}{$P$ value* } \\
\hline & $n$ & $\%$ & $n$ & $\%$ & & & $n$ & $\%$ & $n$ & $\%$ & \\
\hline Sex & & & & & 0.4361 & & & & & & 0.1972 \\
\hline Male & 14 & 29.17 & 135 & 35.53 & & & 128 & 36.16 & 20 & 28.17 & \\
\hline Female & 33 & 70.21 & 245 & 64.14 & & & 226 & 63.84 & 51 & 71.83 & \\
\hline Race & & & & & 0.6988 & & & & & & 0.0175 \\
\hline White & 9 & 18.75 & 63 & 16.54 & & & 66 & 18.54 & 5 & 7.04 & \\
\hline Non-White & 39 & 81.25 & 318 & 83.46 & & & 290 & 81.46 & 66 & 92.96 & \\
\hline Smoking & & & & & 0.1853 & & & & & & 0.1907 \\
\hline Current/former & 9 & 20.00 & 106 & 29.44 & & & 91 & 26.92 & 23 & 34.85 & \\
\hline Never & 36 & 80.00 & 254 & 70.56 & & & 247 & 73.08 & 43 & 65.15 & \\
\hline Insurance coverage & & & & & 0.1780 & & & & & & 0.1322 \\
\hline Private & 17 & 35.42 & 174 & 45.67 & & & 165 & 46.35 & 26 & 36.62 & \\
\hline Public & 31 & 64.58 & 207 & 54.33 & & & 191 & 53.65 & 45 & 63.38 & \\
\hline Cancer $^{\#}$ & & & & & 0.7837 & & & & & & 0.6446 \\
\hline Yes & 3 & 6.25 & 33 & 8.64 & & & 31 & 8.71 & 5 & 7.04 & \\
\hline No & 45 & 93.75 & 349 & 91.36 & & & 325 & 91.29 & 66 & 92.96 & \\
\hline Immunosuppressant use $\mathrm{e}^{\# \#}$ & & & & & 0.9210 & & & & & & 0.0275 \\
\hline Yes & 11 & 22.92 & 90 & 23.56 & & & 77 & 21.63 & 24 & 33.80 & \\
\hline No & 37 & 77.08 & 292 & 76.44 & & & 279 & 78.37 & 47 & 66.20 & \\
\hline AIDS & & & & & $1.000^{+}$ & & & & & & $1.0000^{+}$ \\
\hline Yes & 1 & 2.08 & 9 & 2.36 & & & 9 & 2.53 & 1 & 1.41 & \\
\hline No & 47 & 97.92 & 373 & 97.64 & & & 347 & 97.47 & 70 & 98.59 & \\
\hline Diabetes mellitus & & & & & 0.0449 & & & & & & $<0.0001$ \\
\hline Yes & 6 & 12.50 & 98 & 25.65 & & & 71 & 19.94 & 32 & 45.07 & \\
\hline No & 42 & 87.50 & 284 & 74.35 & & & 285 & 80.06 & 39 & 54.93 & \\
\hline Chronic heart failure & & & & & 0.4928 & & & & & & $0.0629^{+}$ \\
\hline Yes & 1 & 2.08 & 20 & 5.24 & & & 14 & 3.93 & 7 & 9.86 & \\
\hline No & 47 & 97.92 & 362 & 94.76 & & & 342 & 96.07 & 64 & 90.14 & \\
\hline Obstructive lung disease & & & & & 0.0016 & & & & & & $\mathbf{0 . 0 3 5 0}$ \\
\hline Yes & 18 & 37.50 & 69 & 18.06 & & & 66 & 18.54 & 21 & 29.58 & \\
\hline No & 30 & 62.50 & 313 & 81.94 & & & 290 & 81.46 & 50 & 70.42 & \\
\hline Hypertension & & & & & 0.3172 & & & & & & $<0.0001$ \\
\hline Yes & 16 & 33.33 & 156 & 40.84 & & & 127 & 35.67 & 44 & 61.97 & \\
\hline No & 32 & 66.67 & 226 & 59.16 & & & 229 & 64.33 & 27 & 38.03 & \\
\hline Chronic kidney disease & & & & & 0.5592 & & & & & & 0.0209 \\
\hline Yes & 2 & 4.17 & 30 & 7.85 & & & 22 & 6.18 & 10 & 14.08 & \\
\hline No & 46 & 95.83 & 352 & 92.15 & & & 334 & 93.82 & 61 & 85.92 & \\
\hline & $n$ & Mean (SD) & $n$ & Mean & (SD) & $P$ value** & $n$ & Mean (SD) & $n$ & Mean (SD) & $P$ value** \\
\hline Age & 48 & 47.88 (17.09) & 381 & 52.29 & $(16.70)$ & 0.0861 & 356 & $50.18(16.26)$ & 71 & $60.38(16.83)$ & $<0.0001$ \\
\hline BMI & 48 & $30.30(8.56)$ & 367 & 31.47 & $(8.03)$ & 0.3465 & 344 & $30.97(7.95)$ & 69 & $33.20(8.58)$ & 0.0368 \\
\hline
\end{tabular}

Missing values were encountered in $3(0.7 \%)$ for sex, 1 race $(0.2 \%), 25(5.8 \%)$ smoking, $1(0.2 \%)$ insurance status. There were no missing values for immunosuppressant use, cancer diagnosis, AIDS diagnosis, CHF, OLD, Hypertension, CKD

The other skin diseases included onychomycosis $(n=98)$, acne $(n=47)$, actinic keratosis $(n=16)$, allergic contact dermatitis $(n=18)$, alopecia unspecified $(n=52)$, basal cell carcinoma $(n=5)$, cutaneous lupus $(n=2)$, unspecified dermatitis $(n=25)$, dermatomyositis $(n=1)$, condyloma accuminata $(n=8)$, hand dermatitis $(n=7)$, hemangioma $(n=2)$, herpes simplex infection $(n=32)$, herpes zoster infection $(n=8)$, hidradenitis suppurativa $(n=15)$, hirsutism $(n=8)$, hyperhidrosis $(n=10)$, impetigo $(n=2)$, irritant contact dermatitis $(n=8)$, melanoma $(n=1)$, paronychia $(n=1)$, pityriasis rosea $(n=1)$, plantar wart $(n=11)$, psoriasis $(n=11)$, prurigo nodularis $(n=1)$, rosacea $(n=9)$, scabies $(n=1)$, seborrheic dermatitis $(n=39)$, seborrheic keratosis $(n=22)$, squamous cell carcinoma $(n=3)$, tinea $(n=53)$ and urticaria $(n=17)$

Boldface indicates significance 
Table 1 (continued)

${ }^{+}$Fisher exact test used to calculate significance in instances when $25 \%$ of cells had frequencies $<5$

*Chi-squared test

$* * t$ test

${ }^{\#}$ Cancer diagnosis includes solid tumor, leukemia and lymphoma. Specific diagnosis and cancer treatment status not available

\#\# Immunosuppressant drugs ( $n$; \% of total dataset) included adalimumab $(n=4 ; 0.93 \%)$, azathioprine $(n=2 ; 0.47 \%)$, cyclosporine $(n=1 ; 0.23 \%)$, dupilumab $(n=1,0.23 \%)$, etanercept $(n=1,0.23 \%)$, hydroxychloroquine $(n=20 ; 4.65 \%)$, infliximab $(n=3 ; 0.7 \%)$, ixekizumab $(n=1 ; 0.23 \%)$, methotrexate $(n=12 ; 2.79 \%)$, methylprednisolone $(n=8 ; 1.86 \%)$, mycophenolate mofetil $(n=8 ; 1.86 \%)$, prednisone $(n=49 ; 11.4 \%)$, rituximab $(n=1 ; 0.23 \%)$, sirolimus $(n=1 ; 0.23 \%)$, tacrolimus $(n=8 ; 1.86 \%)$, tofacitinib $(n=1 ; 0.23 \%)$, other $(n=7 ; 1.63 \%)$

long-term morbidity) and $\mathrm{AD}$ as the independent variable. Multivariable models adjusted for socio-demographics and comorbidities. Crude and adjusted odds ratio (OR) and 95\% confidence intervals (CI) were estimated.

\section{Results}

Overall, 430 adults were identified with confirmed SARSCoV-2 and a diagnosed skin disease, including 48 (11.2\%) with diagnosed AD. Most (81.25\%) AD patients were nonWhite. There were no significant differences of age, BMI, sex, race, insurance coverage, malignancy or AIDS diagnoses or immunosuppressant use between those with vs. without AD. Patients with vs. without AD had lower rates of diabetes mellitus (DM; 12.50 vs. $25.65 \%, P=0.0449$ ) and higher rates of obstructive lung disease (37.50 vs. $18.06 \%$, $P=0.0016)$. COVID-19 severity was associated with older age, higher BMI, non-White race, immunosuppressant use, obstructive lung disease, hypertension, chronic kidney disease and DM.

Among SARS-CoV-2 positive adult patients, those with vs. without AD had similar COVID-19 clinical outcomes. In fully adjusted models, diagnosed AD had similar odds of hospitalization (adjusted odds ratio [95\% confidence interval]: $0.51[0.20-1.35])$, acute level of care at initial medical care (0.67 [0.35-1.30]), severe-critical SARS-CoV-2 (0.82 [0.29-2.30]), requirement of supplemental non-mechanical oxygen therapy (1.33 [0.50-3.58]), extended hospital stay
(2.24 [0.36-13.85]), lingering COVID-19 symptoms ( 0.58 [0.06-5.31]) and COVID-19 death $(0.002[<0.001->999])$ compared to those without AD. Similar results were observed in unadjusted models (Table 2).

\section{Discussion}

These findings are consistent with studies that found no association of $\mathrm{AD}$ with COVID-19 morbidity. AD patients may be more susceptible to acquiring SARS-CoV-2 infection [3], though findings are inconclusive [5]. Current evidence indicates that $\mathrm{AD}$ patients are not at increased risk of mechanical ventilation [3, 4], hospitalization [2], longer hospital stay [4], intensive care unit admission [4] or death [2, 4]. In one retrospective study, AD was inversely associated with COVID-19 hospitalization [1]. We further demonstrate that $\mathrm{AD}$ is not associated with various other COVID-19 outcomes, including supplemental oxygen therapy, lingering symptoms and acuity level of initial care.

Study strengths include examination of multiple COVID-19 outcomes and controlling for confounders in multivariable analyses. Limitations include small sample size of AD patients, recruitment from a single metropolitan academic center, racial homogeneity and lack of stratified analysis by SARS-CoV-2 variants or AD features. Future studies with larger samples can further elucidate potential associations between $\mathrm{AD}$ and COVID-19. 
Table 2 Association of atopic dermatitis with COVID-19 severity and hospitalization

\begin{tabular}{|c|c|c|c|c|c|c|}
\hline \multirow[t]{2}{*}{ Outcome } & \multicolumn{2}{|c|}{$\begin{array}{l}\text { Atopic dermatitis } \\
n(\%)\end{array}$} & \multirow[t]{2}{*}{$\begin{array}{l}\text { Crude OR } \\
(95 \% \mathrm{CI})\end{array}$} & \multirow[t]{2}{*}{$P$ value } & \multirow[t]{2}{*}{$\begin{array}{l}\text { Adjusted OR } \\
(95 \% \mathrm{CI})\end{array}$} & \multirow[t]{2}{*}{$P$ value } \\
\hline & Yes & No & & & & \\
\hline \multicolumn{7}{|l|}{ Hospitalization ${ }^{\#}$} \\
\hline No & $38(84.44)$ & $259(71.75)$ & 1.00 (ref) & - & 1.00 (ref) & - \\
\hline Yes & $7(15.56)$ & $102(28.25)$ & $0.47(0.20-1.08)$ & 0.0756 & $0.51(0.20-1.35)$ & 0.1772 \\
\hline Visit type $^{\#}$ & & & & & & - \\
\hline Outpatient & $22(45.83)$ & $141(37.11)$ & 1.00 (ref) & - & 1.00 (ref) & - \\
\hline Inpatient & $26(54.17)$ & $239(62.89)$ & $0.70(0.38-1.28)$ & 0.2424 & $0.67(0.35-1.30)$ & 0.2304 \\
\hline \multicolumn{7}{|l|}{ Oxygen therapy\# } \\
\hline No & $41(85.42)$ & $313(83.47)$ & 1.00 (ref) & - & 1.00 (ref) & - \\
\hline Yes & $7(14.58)$ & $62(16.53)$ & $1.07(0.45-2.51)$ & 0.8788 & $1.33(0.50-3.58)$ & 0.5686 \\
\hline \multicolumn{7}{|l|}{ COVID-19 severity ${ }^{\#}$} \\
\hline Asymptomatic-mild & $42(87.50)$ & $314(82.85)$ & 1.00 (ref) & - & 1.00 (ref) & - \\
\hline Severe-critical & $6(12.50)$ & $65(17.15)$ & $0.69(0.28-1.70)$ & 0.4178 & $0.82(0.29-2.30)$ & 0.7062 \\
\hline \multicolumn{7}{|l|}{ Hospital duration ${ }^{\#}$} \\
\hline $1-6$ days & $3(42.86)$ & $59(59.60)$ & 1.00 (ref) & - & 1.00 (ref) & - \\
\hline$\geq 7$ days & $4(57.14)$ & $40(40.40)$ & $1.97(0.42-9.26)$ & 0.3924 & $2.24(0.36-13.85)$ & 0.3857 \\
\hline \multicolumn{7}{|l|}{ Course $\mathrm{H}^{\# \#}$} \\
\hline Recovered & $46(97.87)$ & $338(94.15)$ & 1.00 (ref) & - & 1.00 (ref) & - \\
\hline Chronic complications & $1(2.13)$ & $12(3.34)$ & $0.61(0.08-4.82)$ & 0.6412 & $0.58(0.06-5.31)$ & 0.6328 \\
\hline Death & $0(0)$ & $9(2.51)$ & $<0.001(<0.001->999)$ & 0.9631 & $0.002(<0.001->999)$ & 0.7781 \\
\hline
\end{tabular}

\#Binary logistic regression models were constructed with atopic dermatitis diagnosis as the independent variable and COVID-19 outcomes as the dependent variables. Dependent variables included hospitalization (yes vs. no), visit type (inpatient vs. outpatient), oxygen therapy (yes vs. no), COVID-19 severity (severe-critical vs. asymptomatic-mild) and hospital duration (1-6 days vs $\geq 7$ days)

\#\# Multinomial logistic regression models were constructed with atopic dermatitis diagnosis as the independent variable (yes/no) and COVID-19 course as the dependent outcome variable (chronic complications or death vs recovered). Crude odds ratios (OR) and $95 \%$ confidence intervals (CI) were generated for unadjusted models

Adjusted OR and 95\% CI were generated for age [continuous], sex [male/female], race [White/non-White], immunosuppressant use [yes/no], smoking [current-former/never], BMI [continuous], insurance status [public/private], diagnosis of cancer [yes/no], and AIDS [yes/no]

Author contributions JIS had full access to all the data in the study and takes responsibility for the integrity of the data and accuracy of the data analysis. JIS: study concept and design. JIS, KN, BM, JM, GS, BL, UR, TK, AG: acquisition of data. UR, TK, AG, JIS: analysis and interpretation of data. UR, TK, AG, JIS: drafting of the manuscript. UR, TK, AG, JIS: critical revision of the manuscript for important intellectual content. UR, TK, AG, JIS: statistical analysis.

Funding None.

Data availability Available upon request.

Code availability Available upon request.

\section{Declarations}

Conflict of interest None.

\section{References}

1. Keswani A, Dhana K, Rosenthal JA et al (2020) Atopy is predictive of a decreased need for hospitalization for coronavirus disease 2019. Ann Allergy Asthma Immunol 125:479-481. https://doi.org/10.1016/j.anai.2020.07.012

2. Nguyen C, Yale K, Casale F et al (2021) SARS-CoV-2 infection in patients with atopic dermatitis: a cross-sectional study. Br J Dermatol. https://doi.org/10.1111/bjd.20435

3. Patrick MT, Zhang H, Wasikowski R et al (2021) Associations between COVID-19 and skin conditions identified through epidemiology and genomic studies. J Allergy Clin Immunol 147:857-869. e7. https://doi.org/10.1016/j.jaci.2021.01.006

4. Yang JM, Koh HY, Moon SY et al (2020) Allergic disorders and susceptibility to and severity of COVID-19: a nationwide cohort study. J Allergy Clin Immunol 146:790-798. https://doi.org/10.1016/j.jaci. 2020.08.008

5. Yiu ZZN, Harding-Oredugba G, Griffiths CEM et al (2021) Risk of COVID-19 infection in adult patients with atopic eczema and psoriasis: a single centre, cross-sectional study. Br J Dermatol. https:// doi.org/10.1111/bjd.20062

Publisher's Note Springer Nature remains neutral with regard to jurisdictional claims in published maps and institutional affiliations. 